Supplement of SOIL, 1, 381-397, 2015

http://www.soil-journal.net/1/381/2015/

doi:10.5194/soil-1-381-2015-supplement

(c) Author(s) 2015. CC Attribution 3.0 License.

(c) (i)

Supplement of

\title{
A new synthesis for terrestrial nitrogen inputs
}

B. Z. Houlton and S. L. Morford

Correspondence to: B. Z. Houlton (bzhoulton@ucdavis.edu) 
Supplemental Table: Summary of ecosytem nitrogen accretion values from Binkey et al. (2000) and Johnson et al. (2014). The lithology at each study site was evaluated for potential N enrichment given the type of host rock.

\begin{tabular}{|c|c|c|c|c|c|}
\hline Reference(s) & Location & $\begin{array}{l}\text { Naccretion } \\
\left(\mathrm{kg} \mathrm{ha}^{-1} \mathrm{yr}^{-1}\right)\end{array}$ & Parent Material & $\begin{array}{l}\text { Parent Material N } \\
\text { Potential }\end{array}$ & Notes \\
\hline Bormann et al. (1993) & $\begin{array}{l}\text { Hubbard Brook Experimental Forest } \\
\text { (Sandbox), New Hampshire, USA }\end{array}$ & 60 to 152 & alluvium (low $\mathrm{N}$ ) & No & \\
\hline $\begin{array}{l}\text { Dickson \& Crocker (1953), } \\
\text { Sollins et al. (1983) }\end{array}$ & Mt. Shasta, California, USA & 5 to 60 & $\begin{array}{l}\text { Mudflow (Volcanic: mafic- } \\
\text { intermediate) }\end{array}$ & No & $\begin{array}{l}\text { unknown if mudflows contain } \mathrm{N} \text { from } \\
\text { soils/vegetetation caught up in flow? }\end{array}$ \\
\hline Turner and Lambert (2011) & $\begin{array}{l}\text { Sunny Corner Forest, New South Wales, } \\
\qquad \mathrm{AU}\end{array}$ & 13 to 22 & Volcanic (felsic) & No & $\begin{array}{l}\text { Included only RONOBO and RONOBO } \\
\text { treatments. }\end{array}$ \\
\hline Morrison and Foster (2001) & Turkey Lakes, Ontario, CA & 16 & $\begin{array}{l}\text { Igneous/Meta-igneous (Greestone } \\
\text { and Granite) }\end{array}$ & No & \\
\hline Richter and Markewitz (2000) & Calhoun Forests, South Carolina, USA & 0 to 14 & Granodiorite & No & \\
\hline Bormann \& Likens (1977) & $\begin{array}{c}\text { Hubbard Brook Experimental Forest, New } \\
\text { Hampshire, USA }\end{array}$ & 14 & $\begin{array}{l}\text { high-grade metasedimentary } \\
\text { (amphibolite) }\end{array}$ & No & \\
\hline Alriksson \& Olsson (1995) & Southern Sweden & -18 to 12 & Granite & No & \\
\hline Garcia-Montiel (1996) & Luquillo Experimental Forest, Puerto Rico & -5.7 to 6.2 & Tuff Breccia & No & \\
\hline Aguilera et al. (1993) & $\begin{array}{l}\text { H. J. Andrews Research Research Forest, } \\
\text { Blue River, Oregon, USA. }\end{array}$ & 2 to 3 & Volcanic: mafic-intermediate & No & \\
\hline Johnson et al . (1995) & $\begin{array}{c}\text { Hubbard Brook Experimental Forest, New } \\
\text { Hampshire, USA }\end{array}$ & 0 & $\begin{array}{l}\text { high-grade metasedimentary } \\
\text { (amphibolite) }\end{array}$ & No & \\
\hline Knoepp \& Swank (1997) & $\begin{array}{c}\text { Coweeta Hydrologic Laboratory, North } \\
\text { Carolina, USA }\end{array}$ & -60 to -50 & $\begin{array}{c}\text { high-grade meta-igneous \& } \\
\text { metasedimentary (amphibolite) }\end{array}$ & No & \\
\hline Fisher \& Eastburn (1974) & Illinois, USA & 0 to 110 & $\begin{array}{l}\text { Sedimentary (siliciclastic and } \\
\text { carbonate) }\end{array}$ & Yes & $\begin{array}{l}\text { Parent material inferred from soil } \\
\text { series description in paper }\end{array}$ \\
\hline Johnson \& Todd (1998) & $\begin{array}{c}\text { Walker Branch Watershed, Tennessee, } \\
\text { USA }\end{array}$ & 44 to 87 & $\begin{array}{l}\text { Sedimentary (carbonate and } \\
\text { siliciclastic) }\end{array}$ & Yes & \\
\hline Eriksson \& Rosen (1994) & Susegården, Halmstad, Sweden & 0 to 50 & Till & Yes & \\
\hline Day et al. (1975) & Broadbalk experiment, Rothamsted, UK & 48 & Sedimentary (carbonate/marl) & Yes & \\
\hline Johnson et al. (2007) & $\begin{array}{c}\text { Walker Branch Watershed, Tennessee, } \\
\text { USA }\end{array}$ & -15 to 43 & $\begin{array}{c}\text { Sedimentary (carbonate and } \\
\text { siliciclastic) }\end{array}$ & Yes & \\
\hline Son \& Gower (1992) & $\begin{array}{l}\text { Coulee Experimental Forest, Wisconson, } \\
\text { USA }\end{array}$ & 10 to 40 & $\begin{array}{l}\text { Sedimentary (siliciclastic and } \\
\text { carbonate) }\end{array}$ & Yes & \\
\hline $\begin{array}{l}\text { Turner et al. (2002), Binkley } \\
\text { et al. (2003) }\end{array}$ & $\begin{array}{l}\text { Penrose State Forest, New South Wales, } \\
\qquad \mathrm{AU}\end{array}$ & 16 to 24 & Sedimentary (Siliciclastic) & Yes & \\
\hline
\end{tabular}




\begin{tabular}{|c|c|c|c|c|c|}
\hline Reference(s) & Location & $\begin{array}{l}\text { N accretion } \\
\left(\mathrm{kg} \mathrm{ha}^{-1} \mathrm{yr}^{-1}\right) \\
\end{array}$ & Parent Material & $\begin{array}{c}\text { Parent Material N } \\
\text { Potential }\end{array}$ & Notes \\
\hline $\begin{array}{l}\text { Jorgensen \& Wells (1971), } \\
\text { Binkley et al.(1992) }\end{array}$ & $\begin{array}{l}\text { Westvaco experimental forest, South } \\
\text { Carolina, USA }\end{array}$ & 0 to 23 & alluvium (sedimentary provinance) & Yes & \\
\hline Turner \& Lambert (2013) & Lidsdale State Forest, Sydney, AU & 20.4 & Sedimentary (Siliciclastic) & Yes & \\
\hline Johnson et al (2003b) & $\begin{array}{c}\text { Walker Branch Watershed, Tennessee, } \\
\text { USA }\end{array}$ & 20 & $\begin{array}{c}\text { Sedimentary (carbonate and } \\
\text { siliciclastic) }\end{array}$ & Yes & \\
\hline Guo et al. (2008) & $\begin{array}{c}\text { Kowen Forest, Australian Capital } \\
\text { Territory, AU }\end{array}$ & 17.2 & Sedimentary (Siliciclastic) & Yes & \\
\hline Turner \& Lambert (1986) & $\begin{array}{l}\text { Belango State Forest, New South Wales, } \\
\qquad \mathrm{AU}\end{array}$ & 17 & Sedimentary (Siliciclastic) & Yes & \\
\hline Trettin \& Johnson (2000) & $\begin{array}{c}\text { Walker Branch Watershed, Tennessee, } \\
\text { USA }\end{array}$ & -76 to 6 & $\begin{array}{c}\text { Sedimentary (carbonate and } \\
\text { siliciclastic) }\end{array}$ & Yes & \\
\hline Hopmans \& Elms (2009) & Victoria, Australia & 4.2 & $\begin{array}{c}\text { Sedimentary (carbonate and } \\
\text { siliciclastic) }\end{array}$ & Yes & \\
\hline Kiser (2009) & $\begin{array}{c}\text { Camp Branch Experimental Watershed, } \\
\text { Tennessee, USA }\end{array}$ & 3 & Sedimentary (Siliciclastic) & Yes & \\
\hline Johnson et al. (2003a) & Kennedy Space Center, Florida USA & -32 to -3 & Quaternary alluvium & Yes & \\
\hline $\begin{array}{l}\text { Jenny (1980), Dormar \& } \\
\text { Lutwick (1966) }\end{array}$ & Alberta, Canada & -90 & Sedimentary (Siliciclastic) & Yes & \\
\hline Turvey \& Smethurt (1988) & Victoria, Australia & 0 & unknown & unknown & $\begin{array}{l}\text { reference unavailable (excluded from } \\
\text { plot) }\end{array}$ \\
\hline Bacon et al. (1996) & Whiporie, New South Wales, AU & 10.1 & Unknown & Unknown & $\begin{array}{l}\text { reference unavailable (excluded from } \\
\text { plot) }\end{array}$ \\
\hline Turner \& Lambert (2012) & Lidsdale, New South Wales, AU & 8.5 & Unknown & Unknown & $\begin{array}{l}\text { Either Granite or siliciclastic (excluded } \\
\text { from plot) }\end{array}$ \\
\hline Mroz et al. (1985) & Upper Michigan & -450 & alluvium (sedimentary provinance) & Yes & $\begin{array}{l}\text { Soil Removal drove } \mathrm{N} \text { loss (excluded } \\
\text { from plot) }\end{array}$ \\
\hline
\end{tabular}

